\title{
Effectiveness of Five-day-old 10\% Bleach in a Student Microbiology Laboratory Setting
}

\section{NANCY GOODYEAR}

\section{ABSTRACT}

In the student laboratory, $10 \%$ bleach (sodium hypochlorite) is used to disinfect benches before and after work, and when a spill occurs. Centers for Disease Control and Prevention (CDC) Guidelines recommend diluting bleach daily. In a student laboratory setting, the organisms in use are known and may include only selected standard bacteria. Diluting bleach daily is time consuming and wastes bleach. We hypothesized that $10 \%$ bleach stored in low density polyethylene (LDPE) wash bottles would maintain sufficient chlorine concentration to be effective against the organisms used in the student laboratory for five days, so that bleach could be diluted weekly instead of daily. Approximately $3 \times 10^{6} \mathrm{CFU}$ of each bacterium were spotted to a laboratory bench surface in duplicate and allowed to air dry. One spot was individually cleaned with five-day old $10 \%$ bleach following the same protocol as student laboratories. The second spot was uncleaned and sampled as a control. Contact plates containing D/E Neutralizing agar were touched to the spots, incubated overnight at $35^{\circ} \mathrm{C}$ and examined for growth. An uninoculated spot was also sampled as a background control. A total of 22 different organisms were tested, representing the major groups of organisms used in the student laboratories. All organisms tested were eliminated by the five-day old bleach. All uncleaned spots showed dense growth. The background control had no growth. Reducing the dilution of bleach to once a week rather than daily will save time and money, which can then be devoted to more teaching and curriculum responsibilities, while still maintaining laboratory safety.

\section{ABBREVIATIONS \\ LDPE-low density polyethylene, CDC-Centers for Disease Control and Prevention, CFU-colony forming units, TSA-tryptic soy agar, TSB-tryptic soy broth}

\section{INDEX TERMS}

Disinfection, education, microbiology, sodium hypochlorite

Clin Lab Sci 2012;25(4):219

Nancy Goodyear, PhD, MLS(ASCP) ${ }^{C M}$, University of Massachusetts Lowell, Lowell, MA

Address for Correspondence: Nancy Goodyear, PhD, MLS(ASCP $)^{C M}$, 3 Solomont Way, Suite 4, University of Massachusetts Lowell, Lowell, MA 01854, 978-9344427, Nancy_Goodyear@uml.edu

\section{INTRODUCTION}

Sodium hypochlorite, a chlorine-releasing agent, is a very common disinfectant in healthcare and laboratory settings and has a broad range of antimicrobial activity. ${ }^{1,2}$ The Centers for Disease Control and Prevention (CDC) Guideline for Disinfection and Sterilization in Healthcare Facilities recommends preparing $10 \%$ bleach daily in order to ensure adequate disinfection of infectious agents. ${ }^{1}$ This is standard practice in clinical settings; however, this recommendation is based on the need to kill all potential pathogens, including non-enveloped viruses, in patient care facilities. In the student laboratory setting only known bacteria and yeast are in use, and there may not be any patient samples in use.

The Department of Clinical Laboratory and Nutritional Sciences at the University of Massachusetts Lowell offers multiple laboratory sections of Basic and Clinical Microbiology and Pathology (a required course for students majoring in Medical Laboratory Science, Clinical Sciences, Nutritional Sciences, Nursing, Community Health, and Environmental Health), and Medical Microbiology, required for Medical Laboratory Science majors. In the fall semester of 2011 there were seven sections of Basic and Clinical Microbiology enrolling more than 100 students. In the spring semester of 2012 there were two sections of Basic and Clinical Microbiology and Pathology plus two sections 
of Medical Microbiology.

For all laboratory sections, laboratory benches are disinfected with $10 \%$ bleach before and after work, and when spills occur. Diluted bleach is stored in translucent laboratory wash bottles on the bench tops for easy access by students. Student workers, under the oversight of staff, are responsible for refilling the bottles with diluted bleach. If bleach is diluted daily, leftover bleach in the wash bottles must be discarded. This process is time consuming and wastes bleach.

In addition, bleach is a well-known respiratory irritant and is strongly linked to occupational asthma in healthcare workers, even with exposures limited to once or twice a week. ${ }^{3,4}$ The process of diluting bleach daily exposes staff or students repeatedly, increasing the risk of asthma or other respiratory irritation.

Rutala, et $\mathrm{al}^{5}$ showed that bleach diluted to $5 \%$ in tap water at $\mathrm{pH} 8$, stored for 30 days at room temperature in polyethylene wash bottles contained $83 \%$ of the original free chlorine levels. The remaining free chlorine was adequate to kill Pseudomonas aeruginosa, Staphylococcus aureus, and Salmonella choleraesuis.

Our hypothesis was that five-day old 10\% bleach stored at room temperature in polyethylene wash bottles would effectively kill the bacteria used in the student labs, using the standard student laboratory procedure for disinfecting bench surfaces.

\section{MATERIALS AND METHODS Materials and Bacterial Cultures}

Clorox $^{\oplus}$ Regular Bleach (The Clorox Company, Oakland, CA) was purchased from a local retailer and diluted to $10 \%$ with tap water five days before the experiments were performed. The diluted bleach was stored in a translucent low density polyethylene wash bottle (Thermo Fisher Scientific, Inc, Pittsburgh, PA) at room temperature as it would be in the student laboratory.

Bacterial stock strains were obtained from the American Type Culture Collection (Manassas, VA) or from Lowell General Hospital, Lowell, MA (see Table 1). Stock cultures were maintained on TSA (Difco ${ }^{\text {тм }}$ Tryptic Soy Agar, Becton, Dickinson and Co, Sparks, MD) plates. Overnight growth in TSB (Bacto ${ }^{\text {тм }}$ Tryptic
Soy Broth, Becton, Dickinson and Co, Sparks, MD) was used for bench inoculation for most organisms. Streptococcus gallolyticus and Pasteurella multocida were grown in TSB supplemented with 10\% bovine calf serum (CELLect ${ }^{\circ}$ Calf Bovine Serum, MP Biomedicals, LLC, Solon $\mathrm{OH}$ ). The quantity of bacteria used was estimated using a Thermo Spectronic 20D+ spectrophotometer (Thermo Spectronic, Rochester, NY). D/E Neutralizing agar (Difco ${ }^{\mathrm{TM}} \mathrm{D} / \mathrm{E}$ Neutralizing Agar, Becton, Dickinson and Co, Sparks, MD) was used to determine growth after cleaning bench surfaces with $10 \%$ bleach.

\section{Disinfection Testing Procedure}

The procedure was modified from standard surface disinfection protocols. ${ }^{6,7}$ In brief, a clean laboratory bench surface was rinsed with water to remove any remaining bleach or other disinfectant and sectioned into four inch $\mathrm{x}$ four inch squares using laboratory tape. An overnight broth culture of each bacterium was standardized spectrophotometrically to a $1 \mathrm{McFarland}$ (0.257 A, $\left.-3 \times 10^{8} \mathrm{CFU} / \mathrm{mL}\right)$. Approximately $3 \times 10^{6}$ CFU of each bacterium in $10 \mu \mathrm{L}$ were spotted to a laboratory bench surface in duplicate and allowed to air dry. One spot per bacterium was individually cleaned with five-day old $10 \%$ bleach following the student laboratory protocol (bleach squirted onto surface, wiped with a paper towel and allowed to air dry). The second spot was uncleaned and was sampled as a positive control. Contact plates containing D/E Neutralizing agar were touched to the spots, incubated overnight at $35^{\circ} \mathrm{C}$ in either air or $\mathrm{CO}_{2}$ and examined for growth. An uninoculated spot was also sampled as a background control.

\section{Neutralization Test Procedure}

Neutralization tests were performed to confirm that each organism would grow on D/E Agar and to confirm that the disinfecting activity of the bleach was neutralized and did not continue to kill or inhibit organisms after the contact time on the bench had ended. The procedure followed was modified from a standard disinfectant neutralization protocol. ${ }^{8}$ In brief, the laboratory bench was prepared as described above, but no bacteria were spotted. After treating the bench surface with bleach, D/E plates were touched to the surface. Each organism was subcultured to the $\mathrm{D} / \mathrm{E}$ plate after it was touched to the treated laboratory bench. The plates were incubated overnight and 
examined for growth. The presence of growth indicated the neutralization of the bleach, allowing the organism to grow. This also confirmed the ability of each organism to grow on $\mathrm{D} / \mathrm{E}$ agar, which does not support the growth of some fastidious organisms such as Neisseria gonorrohoeae.

\section{RESULTS}

All organisms listed in Table 1 were eliminated by the five-day old bleach. All uncleaned spots showed dense growth. The background control had no growth. The neutralization tests showed that the D/E agar effectively neutralized the activity of bleach and that each of the organisms listed was able to grow on the $\mathrm{D} / \mathrm{E}$ agar.

Table 1. Organisms tested and results.

\begin{tabular}{llcc}
\hline Organism & ATCC \# & Uncleaned & Cleaned \\
& & CFU & CFU \\
Staphylococcus aureus & 6538 & TNTC & 0 \\
Staphylococcus epidermidis & 12228 & TNTC & 0 \\
Rothia mucilaginosa & 20258 & TNTC & 0 \\
Enterococcus faecalis & 29212 & TNTC & 0 \\
Streptococcus pyogenes & 19615 & TNTC & 0 \\
Streptococcus gallolyticus* & 49147 & TNTC & 0 \\
Escherichia coli & 29214 & TNTC & 0 \\
Klebsiella pneumoniae* & 13883 & TNTC & 0 \\
Enterobacter aerogenes & 13048 & TNTC & 0 \\
Proteus vulgaris & 49132 & TNTC & 0 \\
Serratia marcescens & 8100 & TNTC & 0 \\
Salmonella enteriditis & 13076 & TNTC & 0 \\
Shigella sonnei & 9290 & TNTC & 0 \\
Alcaligenes faecalis & 35655 & TNTC & 0 \\
Pseudomonas aeruginosa & 9027 & TNTC & 0 \\
Pasteurella multocida* & 12945 & TNTC & 0 \\
Listeria monocytogenes* & 7644 & TNTC & 0 \\
Bacillus subtilis & 6633 & TNTC & 0 \\
Saccharomyces cerevisiae & 2601 & TNTC & 0 \\
Candida albicans & 10231 & TNTC & 0 \\
Nocardia asteroides & 19247 & TNTC & 0 \\
Mycobacterium smegmatis & 14468 & TNTC & 0 \\
& & & \\
\hline & & &
\end{tabular}

ATCC: American Type Culture Collection; CFU: Colony Forming Units; TNTC: Too Numerous To Count

* Organisms kindly provided by the microbiology laboratory at Lowell General Hospital, Lowell, MA.

\section{DISCUSSION}

In patient care settings, $10 \%$ bleach is commonly used for cleaning and disinfection. Diluting bleach daily is appropriate in direct patient care settings because any infectious agent may be present, including those that are most resistant to disinfection such as non-enveloped viruses and mycobacteria. The highest level of disinfection is required. This practice is also commonly followed in settings such as the student laboratory where there are no patient samples and the infectious agents in use are well known. In student laboratories, only a small volume of diluted bleach may be used each day, but it is difficult to dilute the exact amount needed. This results in the disposal of leftover one-day old bleach as bottles are emptied and refilled. While bleach is not generally expensive, student laboratories budgets are small and any cost savings is helpful. In addition, there is a labor cost in gathering bleach bottles, emptying them, preparing a fresh dilution, refilling the bottles, and redistributing them to the laboratories.

The stability of diluted bleach depends on a number of factors: $\mathrm{pH}$, exposure to light, initial concentration, and the presence of organic soil such as blood. ${ }^{1,5}$ Organic soil such as blood decreases effectiveness due to chemical reactions with the free chlorine and/or the creation of a physical barrier between the organism and the disinfectant. In a student microbiology laboratory the most likely organic soil that would be mixed with organisms in a spill would be liquid culture media or a reagent such as rabbit plasma. Human blood contamination is possible in the case of a student injury, but less likely. These experiments were performed using bacteria grown in liquid media, modeling a real student laboratory situation.

Another factor in effectiveness is contact time. The CDC guidelines recommend a wet contact time of at least 10 minutes. ${ }^{1}$ Wet contact time refers to the amount of time the surface remains visibly wet. However, for routine cleaning of benches in the student laboratory before and after work, students squirt an unmeasured quantity of bleach onto the bench surface, wipe the surface with a paper towel, and leave. The benchtops will dry well before 10 minutes has passed, usually within one minute. Dried bleach residue remains on the surface, but this is not in compliance with the recommended wet contact time.

The active form of diluted bleach is hypochlorous acid $(\mathrm{HOCl})$; the concentration is generally measured as free available chlorine. Hypochlorous acid breaks down rapidly into hypochlorite ion $\left(\mathrm{OCl}^{-}\right)$, which has little antimicrobial activity. ${ }^{2,9}$ This reaction occurs most rapidly at high $\mathrm{pH}(>9)$. The mechanism of action of sodium hypochlorite is not fully understood. It is 
believed that chlorine inhibits specific biochemical pathways and causes protein denaturation and modification of amino acids within the bacterial cell, which ultimately destroys it. ${ }^{1,2}$ Some research has shown that chlorine-releasing agents significantly damage bacterial DNA. ${ }^{10}$

Undiluted household bleach contains between 5.25\% $(52,500 \mathrm{ppm})$ and $6.15 \%(61,500 \mathrm{ppm})$ sodium hypochlorite. Therefore $10 \%$ bleach will contain at least $5,000 \mathrm{ppm}$ free available chlorine. In the absence of organic soil, diluted bleach will kill organisms at as low as five ppm free available chlorine for many vegetative bacteria, and as high as $5,000 \mathrm{ppm}$ for Clostridium difficile spores (10 minute contact time). ${ }^{1,2}$ Rutala et $\mathrm{a}^{5}$ showed that after 30 days, a 5\% dilution of bleach stored at room temperature in similar containers retains $83 \%$ of free available chorine, while a $50 \%$ dilution retained $47 \%$. (In Rutala's study a $10 \%$ bleach dilution was not tested.) If a $10 \%$ dilution starts at 5,000 ppm, even if only $47 \%$ is retained, more than $2,000 \mathrm{ppm}$ will remain, well beyond the level necessary to kill typical bacteria and yeast used in a student laboratory. ${ }^{1}$

An alternative to liquid bleach sometimes used in clinical settings is pre-packaged bleach wipes. The bleach percentage in these wipes is generally less than $1 \%$, while still maintaining a minimum $5,000 \mathrm{ppm}$. In addition, stabilizers are added to preserve the effectiveness of the bleach for a longer period of time. The manufacturer's stated wet contact time for common bacteria is 30 seconds; the contact time for Candida albicans and Mycobacterium bovis is three minutes. ${ }^{11}$ One limitation of pre-packaged wipes is that if the container is not closed properly or they are not used quickly enough the wipes could dry out, resulting in insufficient wet contact time. Although a viable alternative, the cost of pre-packaged wipes is likely prohibitive in a student laboratory when compared to the cost of household bleach.

In our experiments, each organism tested grew on the $\mathrm{D} / \mathrm{E}$ agar and was completely eliminated by the five-day old bleach. D/E agar is a standard medium known to neutralize the activity of sodium hypochlorite. This medium is used to ensure that the activity of the bleach did not continue during the incubation period. The organisms tested were chosen from the list of organisms used in the Basic and Clinical Microbiology and
Pathology and Medical Microbiology laboratories. The range of organisms tested included representatives of most major groups of organisms: Gram-positive cocci in chains and clusters, enteric Gram-negative rods, nonfermenting Gram-negative rods, fastidious Gramnegative rods, Gram-positive rods, yeast, and mycobacteria.

A limitation of this study is that additional organisms that may be used in a student laboratory, such as Neisseria gonorrhoeae and Haemophilus influenzae, will not grow on D/E agar and therefore couldn't be tested with this experimental design. In addition, organic soils such as blood or other liquid media were not tested, only TSB. If patient samples or other organisms are in use, or for clean up of spills, freshly diluted bleach may be more appropriate.

Reducing the dilution of bleach to once a week rather than daily will save time and money, which can then be devoted to more teaching and curriculum responsibilities, while still maintaining laboratory safety. We have shown that a wide range of organisms commonly used in student laboratories are killed by $10 \%$ bleach that has been stored in translucent wash bottles for five days following routine bench cleaning procedures.

\section{ACKNOWLEDGEMENT}

The author would like to thank Alexander Judge for his assistance in performing the preliminary data collection and pilot runs for this study.

\section{REFERENCES:}

1. Rutala WA, Weber DJ; Healthcare Infection Control Practices Advisory Committee. Guideline for Disinfection and Sterilization in Healthcare Facilities, 2008. Available from http://www.cdc.gov/hicpac/Disinfection_Sterilization/acknowl edg.html. Accessed September 17, 2012.

2. Rutala WA, Weber DJ. Uses of inorganic hypochlorite (bleach) in health-care facilities. Clin Microbiol Rev 1997;10(4):597610.

3. Mirabelli MC, Zock JP, Plana E, Anto JM, Benke G, Blanc $\mathrm{PD}$, et al. Occupational risk factors for asthma among nurses and related healthcare professionals in an international study. Occup Environ Med 2007;64:474-9.

4. Arif AA, Delclos GL. Association between cleaning-related chemicals and work-related asthma and asthma symptoms among healthcare professionals. Occup Environ Med 2012;69:35-40.

5. Rutala WA, Cole EC, Thomann CA, Weber DJ. Stability and Bactericidal Activity of Chlorine Solutions. Infect Control Hospital Epidemiol 1998;19(5):323-77. 
6. ASTM Standard E1153-03 (2010). Standard Test Method for Efficacy of Sanitizers Recommended for Inanimate Non-Food Contact Surfaces. ASTM International, West Conshohocken, PA. 2010.

7. US Environmental Protection Agency Office of Pesticide Programs. Standard Operating Procedure for Germicidal Spray Products as Disinfectants: Testing of Staphylococcus aureus, Pseudomonas aeruginosa, and Salmonella enterica. MB-06-05, Revised, 02-04-10. Available at: http://www.epa.gov/ opp00001/methods/atmpa2z.htm (Accessed September 17, 2012).

8. US Environmental Protection Agency Office of Pesticide Programs. Neutralization Confirmation Procedure for Products Evaluated with the AOAC Use Dilution Method and the
AOAC Germicidal Spray Products as Disinfectants Test (Staphylococcus aureus, Pseudomonas aeruginosa, and Salmonella enterica). MB-17-02, Revised, 03-19-12. Available at: http://www.epa.gov/opp00001/methods/atmpa2z.htm (Accessed September 17, 2012).

9. McDonnell G, Russell AD. Antiseptics and Disinfectants: Activity, Action, and Resistance. Clin Microbiol Rev 1999;12:147-79.

10. Dukan S, Touati D. Hypochlorous Acid Stress in Escherichia coli: Resistance, DNA Damage, and Comparison with Hydrogen Peroxide Stress. J Bacteriol 1996;178: 6145-50.

11. Clorox Professional Products Company. Available from http://www.cloroxprofessional.com. Accessed September 17, 2012.

\section{The UNIVERSITY of VERMONT \\ College of Nursing \& Health Sciences \\ Department of Medical Laboratory \& Radiation Science TT Faculty Position (Open Rank)}

The Department of Medical Laboratory and Radiation Sciences at the University of Vermont is seeking an outstanding individual to make a significant contribution to the medical laboratory science program in the College of Nursing and Health Sciences. This full-time, nine-month, tenure track position is open to PhDs or those with an equivalent doctoral degree focused in the medical laboratory sciences or related area. Possession of an MLS, MT or CLS credential is highly desirable. The successful candidate will have a strong commitment to undergraduate education including advising, and will be an active participant in a PhD program either within the College of Nursing and Health Sciences or on campus. The successful candidate will be positioned to generate extramural funding and implement a sustained program of active research. A strong commitment to diversity, interdisciplinary research, and inter-professional education are essential attributes. Unique opportunities for academic leadership may be available with this position.

UVM's MLS program is accredited by NAACLS and has been in existence for over 40 years. UVM has over 12,000 students with more than 100 undergraduate programs, plus numerous master's and doctoral programs. The College of Nursing and Health Sciences is part of the Academic Health Center at the University of Vermont, in partnership with the College of Medicine and Fletcher Allen Health Care, a tertiary-care hospital and primary care health system. Uniquely, Fletcher Allen Health Care's main hospital and ambulatory care center is contiguous to the UVM campus. The Academic Health Center is an integral part of the University of Vermont's vision to be one of the nation's premier, small public research universities pre-eminent in the study of health and the environment. Burlington, Vermont is located on the shore of beautiful Lake Champlain between the Adirondack and Green Mountain ranges. It has been named a top college town and a top city for overall quality of life. Check our website for more info: www.uvm.edu/ and www.uvm.edu/ $\sim \mathrm{cnhs} / \mathrm{mlrs}$

The University of Vermont recently identified three "Spires of Excellence" in which it will strategically focus institutional investments and growth over the next several years: Neuroscience, Behavior and Health; Complex Systems; and Food Systems. Candidates whose research, scholarship, and/or creative work interests align or intersect with these spires are especially encouraged to apply. More information on the Neuroscience, Behavior and Health spire may be found on the UVM website: http://www.uvm.edu/ -tri .

The University is especially interested in candidates who can contribute to the diversity and excellence of the academic community through their research, teaching, and service. Applicants are requested to include in their cover letter information on how they will further this goal.

Prospective candidates should apply online at www.uvmjobs.com Search for the position using the department name (MLRS). Attach curriculum vitae and letter detailing areas of expertise and professional goals. The position will remain open until filled and review of applications will begin December 14th. All inquiries will remain confidential. For additional information please contact:

Paula Deming, PhD, MT

Search Committee Chair

Department of Medical Laboratory and Radiation Science

University of Vermont

302 Rowell, 106 Carrigan Drive

Burlington, VT 05405

paula.deming@uvm.edu

(802) 656-2506

The University of Vermont is an affirmative action/equal opportunity employer and encourages candidacies of women, people of color and people with disabilities. 